\title{
Des innovations sans innovateur. Le temps long de l'Histoire
}

\author{
Innovation without innovator. The long history
}

\author{
Cédric Perrin ${ }^{1}$ \\ ${ }^{1}$ IDHE.S Evry, Université d'Evry Université Paris-Saclay, France, cp2002@orange.fr
}

\begin{abstract}
RÉSUMÉ. En guise de conclusion des deux volumes de Technologie et Innovation sur les trajectoires d'innovations et d'innovateurs, cet article propose en contrepoint une brève réflexion sur les innovations sans innovateur, les inventions sans inventeur, à l'échelle du temps long de l'histoire, à partir de trois exemples : la néolithisation, le moulin et le conteneur.

ABSTRACT. As a conclusion to the two volumes of Technology and Innovation, which center on the trajectories of innovations and innovators, this article provides a brief reflection, throughout history, on innovations without innovators, inventions without inventors using three examples: Neolithization, the mill and the container.

MOTS-CLÉS. Histoire des techniques, innovation, néolithisation, moulin, conteneur, transports maritimes.

KEYWORDS. history of technology, innovation, Neolithization, mill, container, shipping.
\end{abstract}

Parvenus au terme des deux volumes du dossier consacré aux trajectoires d'innovations et d'innovateurs, il nous a paru intéressant de prendre un peu de recul et de proposer un pas de côté. Ce court article conclusif est une incursion, en forme de contrepoint, dans le monde des inventions sans inventeur, des innovations sans innovateur. Qu'une invention puisse ne pas avoir d'inventeur peut paraître saugrenu de prime abord. Il ne s'agit pas défendre l'idée, tout aussi saugrenue, d'inventions et d'innovations spontanées, nées miraculeusement en somme ou révélées aux hommes. Mais, les travaux d'historiennes et d'historiens, comme ceux de Christine Mac Leod sur James Watt notamment [MAC 07], de François Jarrige sur Joseph Marie Jacquard [JAR 09] ou d'Audrey Colonel dans ce numéro ont montré que les récits d'inventeurs hérö̈ques sont des constructions. A l'inverse, depuis le biface jusqu'au smartphone, l'histoire est pleine d'inventions devenues des innovations plus ou moins importantes sans qu'il soit possible de les attribuer à un inventeur connu et bien déterminé. Sans entrer plus avant dans les détails de chacune d'elle, nous avons retenu ici trois exemples de ces innovations ou grappes d'innovation : la néolithisation, le moulin et le conteneur.

\section{La néolithisation : l'âge des innovations?}

Commençons cette incursion en plaçant le curseur très en amont de l'Histoire, définie par convention comme la période documentée par l'écriture (une autre invention sans inventeur), à la charnière de la préhistoire et de la protohistoire : au Néolithique. A vrai dire le néolithique est sans doute moins une période, avec des bornes chronologiques bien délimitées, qu'un processus qui a pu se produire suivant des temporalités différentes, voire pas du tout en certains points du monde : la néolithisation. Que l'on parle du néolithique ou de néolithisation, la racine - néos - renvoie à l'idée même de nouveauté et d'innovation. Le "néo-lithique», c'est la "nouvelle pierre », celle de la pierre polie, par opposition au paléolithique, l'ancienne - paleo - pierre - lithos-, celle de la pierre taillée, selon une typologie ancienne.

Cette distinction entre un paléolithique et un néolithique a été introduite en 1865 par l'archéologue anglais John Lubbock. Il voulait ainsi scinder en deux le premier âge de la préhistoire dans la tripartition proposée en 1836 par le danois Christian Jürgensen Thomsen : l'âge de la pierre, auquel celui-ci fait succéder un âge du bronze puis un âge du fer [LEH 20]. Cette tripartition avec ses 
dénominations est celle d'un archéologue qui attribua à chacun de ces âges le nom du matériau qu'il trouvait en plus grande quantité sur les chantiers de fouille et dont l'apparition lui parut caractériser une évolution, un nouveau temps. Elle est également indissociable de son propre temps. Elle s'inscrit pleinement dans le contexte intellectuel et culturel d'un XIXè siècle marqué notamment par le positivisme et la confiance dans le progrès technique. Cette division des temps anciens repose sur une lecture évolutionniste de l'histoire dans laquelle l'humanité progresse par l'acquisition de nouvelles techniques. Chacun des trois âges se définit par l'apprentissage et la maîtrise d'une matière plus complexe, la pierre puis des métaux. Chaque période dépasse la précédente en savoirs et savoir-faire. Les innovations scandent ainsi l'histoire des hommes sur un chemin linéaire du progrès. Le néolithique voit le jour dans une période elle-même marquée par de nombreuses innovations techniques qui furent alors qualifiées de «révolution industrielle ». Par un effet de mimétisme, le néolithique apparut dès la fin du XIXè siècle comme une rupture comparable, une « révolution néolithique » selon l'expression que l'archéologue australien Vere Gordon Childe a puissamment contribué à populariser dans l'entre-deux-guerres [CHI 49].

Bien que présenté sous le jour d'un archaïsme, le paléolithique recèle en lui une innovation importante : le travail de la pierre lui-même. A cet égard, peut-être peut-on voir dans le biface la plus ancienne - du moins l'une des plus anciennes - invention [BON 20]. Aussi nouveau et « disruptif » soit-il, un objet ne saurait toutefois à lui seul résumer une aussi longue période, pas même ses techniques. Le néolithique ne se résume pas davantage à une nouvelle façon de travailler la pierre. Si certains ont cru y voir une révolution, c'est parce qu'il vit apparaître de nombreuses innovations : la sédentarisation des populations et les villes (Childe parla aussi de "révolution urbaine »), l'élevage et l'agriculture, l'araire, la céramique et la batterie de cuisine, le fil et les textiles, la roue... Même le métal - qui parut d'abord être propre aux périodes suivantes, dites du bronze et du fer - semble avoir été expérimenté dès le néolithique. La néolithisation est donc ce que l'on nommerait aujourd'hui une grappe d'innovations.

Pourtant, à chacune de ces innovations qui caractérisent la néolithisation, nous serions bien en peine d'associer le nom d'un inventeur, et ce pour plusieurs raisons, au moins deux. Tout d'abord, pour cette période antérieure à l'histoire proprement dite, c'est-à-dire dans des sociétés sans écriture, les archéologues ne disposent d'aucun document qui porte un nom. Ils peuvent déterminer où et quand est apparu telle ou telle innovation; en aucun cas qui en est l'auteur. Mais aussi, ensuite, car de « révolution néolithique » il n'y eut pas. La néolithisation ne fût pas une rupture brutale mais un processus très progressif, lent et nuancé, qui s'est déroulé sur des générations (probablement une centaine) d'hommes et de femmes. Selon l'heureuse formule d'Anne Lehoërff, "l'homme ne se réveille pas un beau matin dans les habits du paysan, il le devient» [LEH 20]. En réalité, les néolithiciens ne savent pas vraiment expliquer l'apparition des nouveautés du néolithique, comment furent inventées ses inventions. Il faut se contenter, avec modestie, d'hypothèses. Sans doute ces innovations furent-elles le fruit d'expérimentations, de tâtonnements, d'imitations, de sélections de ce qui paraissait intéressant et devoir être conservé et ce qui ne l'était pas, ou pas encore bien maîtrisé, voire dangereux, avec son lot d'échecs dont nous ne savons rien puisque ceux-ci n'ont pas laissé de traces. «On» inventa l'agriculture [DEM 17]. De la néolithisation, nous avons hérité une série d'innovations majeures mais qui sont des inventions sans inventeurs.

\section{Le moulin comme archétype de l'invention sans inventeur}

Qu'il soit hydraulique ou éolien, à eau ou à vent, le moulin a été la principale source d'énergie, avec la force humaine ou animale, et le premier moteur auxiliaire des activités humaines jusque tard dans le XIXè siècle et le développement des énergies fossiles [BEN 20], avant de connaître un regain d'intérêt comme source d'énergie renouvelable depuis la fin du XXè siècle [BRU 20]. Le moulin fut donc un objet technique banal et des plus répandus, soit une innovation majeure. A ce titre, le moulin apparaît aussi comme l'archétype de l'invention sans inventeur. Son développement présente plusieurs analogies avec le processus de néolithisation. 
Se lancer dans une quête des origines, à laquelle les «molinologues », historiens de métier ou non, ont généralement renoncé après avoir échafaudé diverses hypothèses, serait assez vain. On ne retiendra que quelques repères. Le moulin à eau est sans doute né dans l'orient méditerranéen, dans l'Empire romain, au cours du premier siècle avant notre ère [BLO 35]. Il s'est lentement diffusé vers l'occident puis en Europe du Nord et de l'Est au cours des premiers siècle du Moyen âge. Il faut se garder ici de la tentation du diffusionnisme. Le moulin n'est pas une invention « occidentale » qui aurait essaimé depuis ce foyer originel vers le reste du monde. Sa présence est attestée également dans les mondes indiens et chinois dès la période médiévale. Tout comme la néolithisation, le moulin est très probablement né en plusieurs points du monde, de façon plus ou moins simultanée.

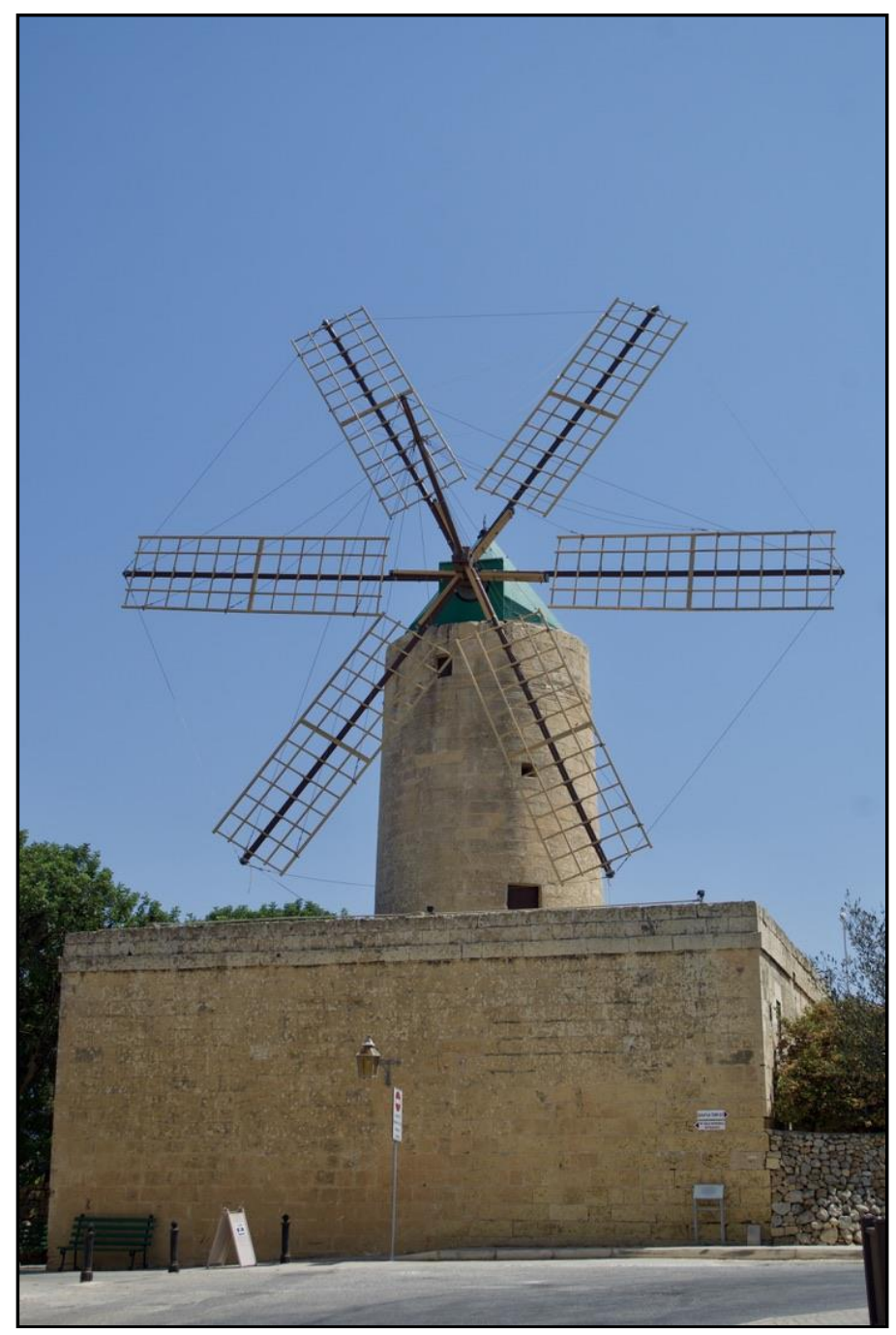

Figure 1. Moulin à vent de Ta' Kola, Xaghra, Gozo, Malte (photographie de l'auteur)

Le moulin à vent est plus tardif. Son histoire fut longtemps délaissée par les historiens des techniques qui n'y voyaient qu'un sous-produit du moulin à eau auquel «on » aurait rajouté des ailes. Il apparaît, du moins en Europe, au XIIè siècle mais lui aussi de façon autonome dans d'autres points du monde. En Perse notamment, il est connu dès le VIIè siècle dans la région très ventée du Sistan (aujourd'hui à cheval sur l'Iran et l'Afghanistan) mais sous la forme particulière d'un moulin vertical dont les pâles se trouvaient insérées dans un bâtiment ouvert aux vents et non à l'extérieur sur un axe horizontal. Ce type de moulin est attesté également en Chine. A partir du XIXè siècle, certains auteurs ont avancé que le moulin à vent aurait transité d'Orient vers l'Occident, via l'Afrique du Nord et l'Espagne, en suivant la conquête arabe. D'autres lui ont prêté une origine nordique. Plus sûrement, le moulin à vent semble se développer pour subvenir aux besoins énergétiques alors que les rivières d'Europe de l'Ouest étaient déjà largement équipées en moulins à eau. Vers la fin du XVè siècle, près de 72000 moulins - 60000 à eau, 12000 à vent - auraient été en action, rien qu'en France [ROB 82]. L'estimation doit sans doute être retenue comme un ordre de 
grandeur mais elle donne une idée de la diffusion et de la banalisation des moulins dans les paysages de l'Europe médiévale.

Tout comme pour les inventions du néolithique, historiens et archéologues sont réduits à des conjectures et des hypothèses sur le pourquoi et le comment. Il a peut-être servi d'abord pour puiser l'eau, l'irrigation, avant de moudre les grains. Il aurait alors été développé dans le sillage de l'agriculture. Les moulins à vent se sont également développés pour des usages hydrauliques, le drainage, relever l'eau des canaux, par exemple en Flandres. Les moulins ont aussi servi à une myriade d'activités industrielles telles que fouler des draps, broyer les écorces utilisées dans le tannage ou des chiffons pour le papier, actionner des marteaux et des soufflets de forges, des scies ou encore des meules pour affuter des outils... [LAN 04]. Ils servent également à écraser des graines pour fabriquer de l'huile. L'étude de ces moulins a ainsi fortement contribué à réévaluer les technologies médiévales. Le moulin fut la machine des industries médiévales, voire, pour certains auteurs comme Lynn White et Jean Gimpel, le support d'une révolution industrielle au Moyen âge.

On ne cherchera pas ici à trancher sur la dénomination de révolution industrielle, et au fond, peu importe ; le moulin est bien une innovation majeure de la période médiévale. En revanche, il est certain que, contrairement à son éponyme du XIXè siècle, celle-ci ne s'est pas accompagnée de la construction d'un récit mettant en valeur de géniaux inventeurs. Le moulin reste en somme une technologie sans inventeur. Il apparaît très fréquemment dans l'iconographie médiévale mais ce sont d'abord les sources matérielles et archéologiques qui permettent aux historiens des techniques de le connaître. L'écrit n'intervient qu'en appui, avec des textes souvent plus tardifs. Des inscriptions sur les traces matérielles, par exemple un axe du moulin de Malderen dans le Brabant (Belgique), révèlent parfois une date, mais pas de noms. Le moulin est un artefact qui contraint l'historien à la modestie, à rompre avec les modèles donnant trop de place aux innovations dites « disruptives » ou radicales, avec l'idée de révolution technique, avec le récit des inventeurs isolés et héroïques. Son histoire tient davantage de l'innovation collective décrite par Robert Allen à partir du cas des hautsfourneaux du nord de l'Angleterre au XIXè siècle [ALL 83]. La rupture technologique cède le pas aux expérimentations qui s'étalent sur un temps long, aux progrès à petits pas, aux améliorations modestes, à la substitution d'un composant, d'un matériau par un autre, à l'ajout d'une pièce, à l'innovation par cran (ou incrémentale) [BRU 20]. Bref, à une autre façon d'aborder l'histoire des techniques et des innovations.

\section{Le conteneur: une révolution des transports maritimes ?}

Les deux situations précédentes pourraient laisser penser qu'il s'agit essentiellement d'un problème de sources relatif à des périodes anciennes pour lesquelles les documents écrits sont rares, sinon absents. Prenons alors pour terminer un événement récent. Le 23 mars 2021 le porteconteneurs Ever-Given s'est retrouvé bloqué en travers du canal de Suez qu'il obstrua du même coup, provoquant un embouteillage maritime monstrueux (plus de 400 navires durent patienter aux entrées du canal). Le fait a abondamment été couvert par les médias internationaux. Les commentateurs ont ironisé sur cette «sardine » qui bloquait le canal ou encore sur le malheureux et maladroit capitaine du navire requalifié en « capitaine de pédalo » et «champion du monde qui a lui tout seul a réussi à bloquer le commerce mondial $»^{1}$. Le fait divers retient l'attention car il condense quelques caractéristiques du capitalisme contemporain : la mondialisation des chaînes de valeur et la croissance des échanges commerciaux mondiaux, la maritimisation de ces échanges dont $80 \%$ se font par la voie maritime (et même $90 \%$ pour les échanges intercontinentaux), l'importance géostratégiques des canaux et des détroits sur les routes maritimes (le canal de Suez voit passer 12 à

\footnotetext{
${ }^{1}$ Olivier de Lagarde, Les Informés, France Info, émission du 27 mars 2021.
} 
13\% du commerce mondial ; il a été emprunté par 18829 bateaux en 2020 selon la Lloyd's) et enfin le rôle des conteneurs et des porte-conteneurs de plus en plus grands (l'Ever-Given fait 200000 tonnes pour $400 \mathrm{~m}$ de long et $59 \mathrm{~m}$ de large), soit une évolution technique dans laquelle certains auteurs n'ont pas manqué de voir une véritable révolution des transports maritimes.

Au premier abord, le conteneur semble avoir un inventeur bien connu : Malcom McLean. A en croire une communication d'entreprise bien huilée et paresseusement ou complaisamment, c'est selon, reprise par de nombreux sites et divers documents, c'est lui qui un beau jour de 1956 ou 1963, voire dès les années 1930, selon les récits, lassé de perdre du temps dans les ports où ses camions se trouvaient immobilisés pour la durée des opérations de déchargement, aurait eu l'idée de détacher les remorques de leur châssis pour les embarquer directement sur le bateau. Le conteneur était né. Ce schéma narratif reprend les lieux communs, qui accompagnent bien des histoires d'invention, de la découverte fortuite, de la fulgurance, de l'éclair de génie d'un individu...

Dans les faits l'histoire est un peu plus compliquée que dans cette narration amplement diffusée. Tout d'abord, McLean ne fut pas le premier à avoir l'idée de transporter des marchandises par bateau dans des boîtes, ni le seul. Dès l'entre-deux-guerres, des transporteurs expérimentèrent le procédé mais il ne connut pas un grand succès. La Grande dépression entraina une contraction des échanges mondiaux peu propice au trafic maritime intercontinental. De plus, les matières premières, agricoles et minérales, constituaient encore la majorité de ces flux et les cargos vraquiers faisaient alors très bien l'affaire. L'usage des boîtes resta cantonné pour l'essentiel à la manutention des valises de passagers dans les voyages transatlantiques. Ces débuts contrariés du transport par conteneur rappellent que pour qu'une invention fasse innovation, il faut qu'elle rencontre un besoin ou parvienne à en créer un, qu'elle trouve un public et une utilité [CHO 17]. A défaut, elle a toutes les chances de vivoter et d'échouer.

Au début des années 1950, McLean, entrepreneur de transports routiers aux Etats-Unis depuis la fin des années 1930 qui connaissait certainement ces précédents, reprit l'idée [LEV 16]. En 1956, il fit aménager le pont de deux anciens pétroliers pour recevoir des remorques de camions débarrassées de leurs essieux [CUD 06]. Pour autant, son entreprise ne connut pas non plus un succès immédiat et fulgurant. Pendant une dizaine d'années, ses conteneurs ne furent en service que le long des côtes états-uniennes ; des armateurs européens en utilisaient aussi le long de leurs propres littoraux sur de courts trajets. Ils étaient également utilisés dans le transport ferroviaire. Ils attendirent encore une dizaine d'années avant de traverser l'Atlantique, puis le Pacifique. De plus, ces «box» ne ressemblaient pas encore non plus aux conteneurs qui se développèrent ensuite. Plusieurs obstacles restaient à lever : la méfiance des syndicats de dockers avec qui il fallut négocier ; la conception et la construction de bateaux (et de wagons) adaptés et l'aménagement des ports qui demandaient de lourds investissements qui ne se firent pas en un jour, ni s'en avoir préalablement convaincus de leur intérêt ; la standardisation de la taille et du volume des conteneurs sans laquelle ceux-ci perdent une grande partie de leur intérêt logistique... Il fallait aussi, sinon avant tout, des marchandises à mettre dans ces boîtes pour les transporter outre-mer.

McLean n'est donc assurément pas le seul acteur de l'histoire et du succès du conteneur. Son apport tient peut-être avant tout à un coup commercial qui assura la croissance de son entreprise. A l'occasion de la Guerre du Vietnam, il parvint à convaincre l'armée américaine de l'intérêt du procédé pour transporter rapidement du matériel en grande quantité et rapidement par la voie maritime. Dans les années suivantes, ce contrat représenta à lui-seul près de la moitié des bénéfices de sa société, la Sea-Land Services, Inc. Dans la marine commerciale, la conteneurisation ne se développa vraiment qu'à partir des années 1970-1980. Elle est intimement liée à l'histoire de la mondialisation et à la libéralisation des échanges commerciaux après la Seconde Guerre mondiale. Les flux de biens manufacturés deviennent alors plus importants que ceux des matières brutes. Alors qu'ils ne représentaient encore qu'un tiers des échanges internationaux de marchandises au début du XXè siècle, ils en ont atteint la moitié au cours des années 1960 puis les trois-quarts dans la 
première décennie du XXIè siècle. Le conteneur a alors été une réponse appropriée à la croissance de ce type d'échanges. En Europe, en Amérique du Nord, en Asie orientale, des ingénieurs, des techniciens et des ouvriers ont travaillé à définir des normes standards (l'équivalent vingt pieds) ; concevoir, améliorer et construire plusieurs générations successives de porte-conteneurs toujours plus grands - depuis les Panamax des années 1980, conçus pour passer les écluses du canal de Panama, aux géants actuels comme l'Ever Given - mais aussi les grues, portiques, chariots, indispensables à la manutention des conteneurs ; aménager les infrastructures du transport maritime (bassins en eau profonde, élargissement ou doublement des canaux, terre-pleins gagnés sur la mer à Singapour, Tokyo, Le Havre et ailleurs)...

Il a donc fallu un bon demi-siècle (de l'entre-deux-guerres aux années 1970-1980), de nombreux aménagements, une suite d'adaptations techniques, un essor et une transformation du commerce maritime mondial, différents acteurs - dont McLean mais aussi de nombreux autres dont l'histoire n'a pas retenu les noms - , pour que la «révolution» des transports maritimes, ou du moins la conteneurisation, prenne corps.

\section{Conclusion}

Le XIXè siècle et sa révolution industrielle ont inventé un âge des inventeurs héroïques qui permettait de légitimer celle-ci et les nouveaux pouvoirs qui ont émergé avec elle [MAC 07]. Mis en perspective sur le temps long de l'histoire, cet âge apparait plutôt comme une parenthèse des inventeurs. L'innovation relève plus souvent d'une expérience collective, étendue sur des temporalités et en suivant des processus qui l'éloignent de l'idée de révolution ou de rupture et, au final, difficile à attribuer à un seul individu. Relativiser le rôle attribué à quelques inventeurs mythifiés dans des récits construits ne doit pas conduire pour autant à nier le rôle des individus dans une histoire sèche et désincarnée au risque d'une certaine forme de déterminisme. Simplement, ces individus doivent être replacés dans des collectifs d'hommes et de femmes qui produisent les innovations, de «travailleurs de l'innovation» [BOI 16]. Revenir sur l'histoire de l'innovation permet de démythifier, de déconstruire les récits lisses du storytelling et des mises en scène flatteuses de la communication d'entreprise auxquels les nouvelles technologies de la communication, tout comme la vogue éditoriale de «l'histoire des marques», ont donné une nouvelle audience. Plus globalement, c'est aussi s'interroger sur les fondements de la valorisation contemporaine du concept d'innovation, devenu omniprésent, y compris dans des champs parfois inattendus. Dans une certaine mesure, l'évolutionnisme du XIXè siècle survit aujourd'hui dans la formule du solutionnisme technologique : l'idée, la conviction même, que nos problèmes contemporains, en particulier la crise écologique et le réchauffement climatique global, pourraient trouver une solution grâce aux innovations techniques [COM 19]. Il ne s'agit pas de négliger l'importance de l'innovation pour l'activité économique et pour les entreprises à qui elle permet d'améliorer leur offre et de durer. Kodak, que nous avons vu dans ce dossier, s'est retrouvée en grande difficulté après avoir raté le passage à la photographie numérique. Il est toutefois permis de se demander si ce n'est pas attendre de la technique plus qu'elle ne peut et si réduire nos problèmes à leur seule dimension technique n'est pas une voie pour éviter une remise en cause plus globale de notre organisation économique, la dernière ruse de la raison, la dernière des milles peaux du capitalisme [DAR 15] pour durer. La seule solution de l'innovation ne risque-t-elle pas dès lors de n'être qu'une illusion?

\section{Références bibliographiques}

[ALL 83] ALLEN Robert, «Collective invention », Journal of Economic Behavior and Organization, n4, 1983.

[BEN 20] BENOIT Serge, D'eau et de feu : forges et énergie hydraulique. XVIII ${ }^{e}$-XX $X^{e}$ siècle. Une histoire singulière de l'industrialisation française, Presses universitaires de Rennes, Rennes, 2020. 
[BOI 16] BOISSARD P., DIDRY C., YOUNES D. (dir.), 2016, Les travailleurs de l'innovation, Presses universitaires de Rennes.

[BLO 35] BLOCH Marc, «Avènement et conquête du moulin à eau », Annales d'histoire économique et sociale, $\mathrm{n}^{\circ} 36$, p. 538-563, 1935.

[BON 20] BON François, «Le biface », dans SINGARAVELOU Pierre, VENAYRE Sylvain, Le magasin du monde, Fayard, Paris, p. 135-139, 2020.

[BRU 20] BRUYERRE Philippe, La puissance du vent. Des moulins à vent aux éoliennes modernes, Presses universitaires du Midi, Toulouse, 2020.

[CHI 49] CHILDE Vere Gordon, L'aube de la civilisation européenne, Payot, Paris, 1949 (pour l'édition française).

[CHO 17] CHOUTEAU Marianne, FOREST Joëlle, NGUYEN Céline, «Quand la culture d'innovation fait écran à la culture technique », Technologie et innovation, $\mathrm{n}^{\circ} 2,2017$.

[COM 19] COMPAGNON Daniel, SAINT-MARTIN Antoine (dir.), «La technique y pourvoira », Socio, n $12,2019$.

[CUD 06] CUDAHY Brian, Box Boats: How Container Ships Changed the World, Fordham University Press, Bronx, New York, 2006.

[DAR 15] DARD Olivier, DIDRY Claude, LE BOT Florent, PERRIN Cédric, «Les mille peaux du capitalisme », L'Homme et la Société, n’ 193/194 \& n’195/196, 2014 \& 2015.

[DEM 17] DEMOULE Jean-Paul, Les Dix millénaires oubliés qui ont fait l'histoire. Quand on inventa l'agriculture, la guerre et les chefs, Fayard, Paris, 2017.

[JAR 09] JARRIGE François, «Le martyre de Jacquard ou le mythe de l'inventeur héroïque (France, XIX ${ }^{\mathrm{e}}$ siècle) », Tracés. Revue de Sciences humaines, n 16, pages 99-117, 2009.

[LAN 04] LANGDON John, Mills in the medieval economy, England 1300-1540, Oxford University Press, Oxford, 2004.

[LEH 20] LEHOERFF Anne, Le néolithique, PUF, Collection « Que sais-je ? », n 4188, Paris, 2020.

[LEV 16] LEVINSON Marc, The Box: How the Shipping Container Made the World Smaller and the World Economy Bigger, Second Edition, Princeton University Press, 2016.

[MAC 07] MAC LEOD Christine, Heroes of Invention. Technology, Liberalism and British Identity, 1750-1914, Cambridge University Press, Cambridge, 2007.

[ROB 82] ROBERT Philipe, «Les premiers moulins à vent », Annales de Normandie, n 32/2, p. 99-120, 1982. 